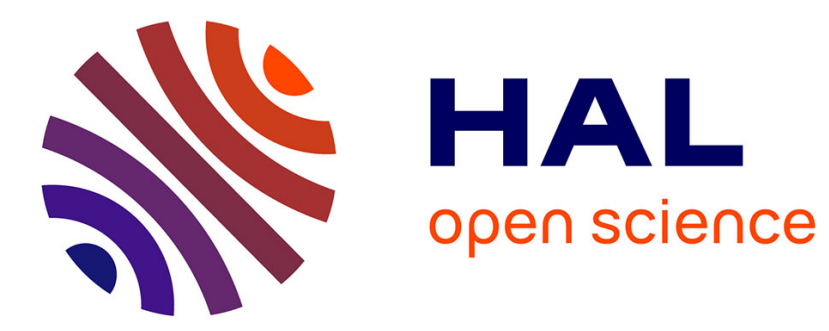

\title{
Le marqueur discursif finnois -hAn: double repérage énonciatif
}

Outi Duvallon

\section{To cite this version:}

Outi Duvallon. Le marqueur discursif finnois -hAn: double repérage énonciatif. Faits de langues, 2014, 43/2014, pp.87-114. hal-01103030

\section{HAL Id: hal-01103030 \\ https://hal.science/hal-01103030}

Submitted on 13 Jan 2015

HAL is a multi-disciplinary open access archive for the deposit and dissemination of scientific research documents, whether they are published or not. The documents may come from teaching and research institutions in France or abroad, or from public or private research centers.
L'archive ouverte pluridisciplinaire HAL, est destinée au dépôt et à la diffusion de documents scientifiques de niveau recherche, publiés ou non, émanant des établissements d'enseignement et de recherche français ou étrangers, des laboratoires publics ou privés. 


\title{
Le marqueur discursif finnois $-h A n$ : double repérage énonciatif
}

\author{
Outi Duvallon*
}

\section{INTRODUCTION}

Le finnois possède un certain nombre de particules enclitiques qui fonctionnent comme marqueurs discursifs : dépourvus de valeur référentielle, prédicative ou grammaticale, ces éléments servent à commenter l'introduction dans le discours d'un segment correspondant à leur portée (cf. Bonnot, 2002). Il s'agit d'unités de la langue dont la description se montre problématique dans la littérature : soit leur identité fait l'objet d'une définition floue et peu opératoire, soit on trouve pour la même unité des définitions fort différentes, parfois contradictoires.

C'est le cas de la particule - $h A n$ à laquelle on s'intéressera ici. D'après ISK ( «La Grande grammaire du finnois», 2004, § 830), -hAn dote l'énoncé d'une nuance selon laquelle son contenu fait partie des connaissances partagées du locuteur et de ses interlocuteurs. Larjavaara $(2007$, p. 436-439, 442), en revanche, considère - $h A n$ comme un élément à valeur épistémico-interactionnelle hautement polysémique, susceptible de produire des effets de sens très divers, tels que surprise, contraste avec l'attente, rappel, appel aux connaissances partagées, justification, objection, insistance, concession, dédramatisation, etc.

Dans cet article, on se propose d'inscrire l'examen de - $h A n$ dans le cadre de la problématique des éléments polycatégoriels. En effet, l'enclitique $-h A n^{1}$ est étymologiquement issu d'un pronom de troisième personne hän «il/elle», et plus précisément des emplois logophoriques (Hagège, 1974) que ce pronom a connus (et connaît toujours) dans les variétés non standard du finnois ${ }^{2}$ (Laitinen, 2002 ; 2005). Notre hypothèse est alors que le marqueur discursif -hAn est porteur d'une

* INALCO/USPC, CNRS UMR8202 IRD UMR135, SeDyL, Courriel : outi.duvallon@inalco.fr

Ce travail est en relation avec l'opération GD3 A cross-linguistic approach of discourse markers de l'Axe 3 du Labex EFL (financé par ANR/CGI). Nous tenons à remercier Christine Bonnot, Auli Hakulinen, Lea Laitinen et Denis Paillard ainsi que les relecteurs anonymes de la revue pour leurs précieuses remarques et suggestions sur une version antérieure du présent article.

${ }^{1}$ Les mots finnois sont soumis à une harmonie vocalique : la lettre capitale $A$ est utilisée pour marquer une voyelle qui peut être soit antérieure ( $\ddot{a}[æ])$, soit postérieure (a $[a])$.

${ }^{2}$ Le processus de standardisation qui a commencé au XIX ${ }^{\mathrm{e}}$ siècle a ignoré la valeur logophorique du pronom hän (voir par ex. Laitinen, 2004, p. 251-252) : dans le finnois standard moderne, la fonction de ce pronom est de référer à un être humain, alors qu'un autre pronom, se «il, elle, ce» sert à désigner des référents non humains. 
identité sémantique abstraite qu'il partage avec le logophorique hän. L'objectif est de s'interroger, d'une part, sur cette identité et de proposer, d'autre part, une définition de la fonction de $-h A n$ en tant que marqueur discursif.

Pour examiner ces deux questions, nous nous situerons dans le prolongement des travaux de Bonnot (2002), Paillard (2009 ; 2011) et Paillard \& Vu Thi (2012) qui considèrent la catégorie des marqueurs discursifs (nommés aussi mots $d u$ discours) comme une partie intégrante des opérations énonciatives qui constituent un dire. La notion clé est celle de scène énonciative (Paillard, 2009) qui est fondée sur l'idée qu'un énoncé $\mathbf{p}$ qui met en mots un état de choses $\mathbf{Z}$ n'est pas le seul énoncé possible, mais toujours dans un rapport de concurrence avec d'autres énoncés, notés p'. Les marqueurs discursifs se définissent comme des mots ou des locutions qui participent à la construction d'un énoncé en y introduisant des déterminations sur le statut de celui-ci dans la scène énonciative, par rapport à différentes composantes de celle-ci (par ex. relation entre $\mathbf{p}$ et $\mathbf{Z}$ ou entre $\mathbf{p}$ et $\mathbf{p}$ ', espace intersubjectif, vouloir dire des mots, vouloir dire du locuteur ou d'un autre sujet, etc.).

La particule - $h A n$ est à l'origine un élément appartenant aux dialectes orientaux du finnois (L. Hakulinen, 1999[1951], p. 46 ; Kiuru, 1990, p. 282 ; Forsman Svensson, 2003, p. 52 ; Forsberg, 1998, p. 295). Les données sur lesquelles s'appuie cet article sont tirées d'un roman intitulé $J u h a^{3}$ publié en 1911 par Juhani Aho (1861-1921), auteur originaire de l'Est de la Finlande. Le corpus relevé dans cette œuvre, avec en tout 297 occurrences de $-h A n$, s'est révélé particulièrement intéressant du point de vue de la question de la polycatégorie, vu le nombre important de cas (plus de $10 \%$ des occurrences) permettant d'observer le passage de $h A n$ d'une catégorie à l'autre 4 .

Cet article s'organise comme suit. Nous commencerons par examiner les propriétés énonciatives du logophorique hän afin d'introduire l'idée d'un double repérage qui caractérise les énoncés en - $h A n$ (partie 2). Ensuite, nous analyserons l'emploi de la particule $-h A n$ dans différents contextes discursifs en prêtant une attention particulière à deux questions : la valeur désassertive de l'énoncé (partie 3 ) et l'accessibilité de la relation prédicative qui est évoquée (partie 4).

\section{ESPACE ÉNONCIATIF SECOND ET REPÈRE VIRTUEL $S_{X}$}

Le logophorique hän «il/elle» à partir duquel le marqueur discursif - $h A n$ s'est développé (Setälä, 1883, p. 92 ; Latvala, 1895, p. 48 ; Ojansuu, 1922, p. 70 ;

\footnotetext{
3 Traduit en français par Lucie Thomas sous le titre L'écume des rapides et publié en 1978 chez Publications Orientalistes de France. La traduction des exemples que nous citons ici s'écarte par endroits de la traduction publiée.

${ }^{4}$ Nous avons constitué aussi un corpus de comparaison, recueilli dans des sources littéraires datant aussi bien de la fin du XIX ${ }^{\mathrm{e}}$ siècle que de la période contemporaine (début du XXI ${ }^{\mathrm{e}}$ siècle), comprenant 787 occurrences de $-h A n$, qui nous a aidée à dégager les contextes d'emploi les plus typiques de ce marqueur discursif.
} 
Forsberg, 1998, p. 295 ; Laitinen, 2002) $)^{5}$ appartient à un système pronominal qui permet de distinguer plusieurs espaces énonciatifs. Le contexte d'emploi typique de hän est celui du discours rapporté, introduit par un verbe du type «dire», comme en (1a) :

(1a)

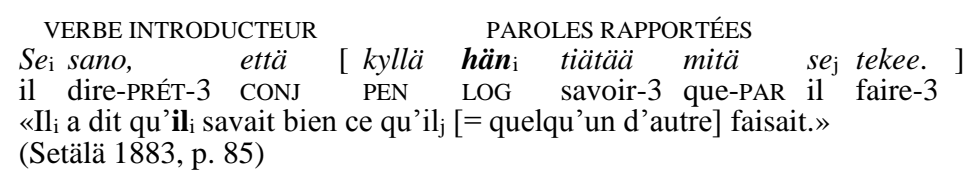

(1b)

$$
\begin{aligned}
& \text { PAROLES DIRECTES } \\
& \text { Kyllä minä tiedän mitä } \\
& \text { PEN je se tekee. } \\
& \text { «Je sais bien ce qu'ili fait.» que-PAR il faire-3 }
\end{aligned}
$$

Situé au sein des paroles rapportées, le logophorique hän «il(/elle)» réfère à l'auteur de ces paroles et s'interprète comme coréférent avec le sujet du verbe introducteur, réalisé par un pronom antilogophorique, $s e_{\mathrm{i}}$ «il(/elle)». Dans cet exemple, le passage - réel ou fictif - du discours direct vers les paroles rapportées consiste à substituer hän au pronom de première personne minä «je» (comparer à ce titre 1a et $1 \mathrm{~b}$ ) afin de désigner un locuteur secondaire, celui de la situation rapportée (cf. Hagège, 1974 ; Roncador, 1992 ; 2006 ; Culy, 1994 ; Stirling, 1994 ; Nau, 2006 ; Boyeldieu, 2013). Le deuxième pronom sujet dans les paroles rapportées, qui est l'antilogophorique $s e_{\mathrm{j}}$ «il(/elle)», marque la disjonction référentielle avec les deux pronoms précédents ${ }^{6}$.

Un autre cas de figure possible, quoique moins fréquent que le premier (Setälä, 1883 , p. 88 ; Latvala, 1895 , p. 44 ; 1899, p. 39, 68 ; Makkonen, 1967, p. 79-84; Kuiri, 1984, p. 122-123), est illustré par l'exemple (2), où le verbe introducteur est kysyä «demander» :

$$
\begin{array}{lllll}
s e_{\mathrm{j}} & \text { kysyi } & \text { is äl } t_{\mathrm{i}} \quad \text { sit } & \text { osaak här } & \text { huattii } \\
\text { il demander-PRÉT-3 } & \text { père-ABL ensuite } & \text { savoir-3-Q LOG } & \text { suédois-PAR } \\
\text { «ensuite } \mathrm{il}_{\mathrm{j}} \text { a demandé à mon père } \text { s'il }_{\mathrm{i}} & \text { parlait suédois» (SMS, s.v. hän) }
\end{array}
$$

Cette fois, le logophorique hän «il» réfère au destinataire des paroles rapportées ${ }^{7}$. La coréférence entre hän et le sujet du verbe introducteur, observée

\footnotetext{
${ }^{5}$ Cf. L. Hakulinen (1979, p. 235-236) et Larjavaara (2007, p. 436) qui mettent en avant le lien entre hän et $-h A n$ sans prendre en considération la logophoricité du premier.

${ }^{6}$ Mis à part le logophorique et la forme verbale à la troisième personne, le «discours rapporté» ne diffère pas des paroles directes (cf. Latvala, 1899, p. 67-68 ; Makkonen, 1967, p. 43-44 ; Kuiri, 1984, p. 116-121 ; Laitinen, 2005, p. 85 ; Roncador, 2006, p. 313). Notons en particulier l'absence de concordance des temps entre le présent du discours rapporté et le passé du verbe introducteur. De même, les marqueurs déictiques et énonciatifs, comme en (1a) la particule énonciative kyllä qui sert à appuyer la polarité affirmative du verbe (cf. Hakulinen, 2001a), relèvent de la situation d'énonciation des paroles présentées.

7 Cf. logophoric addressee pronoun chez Huang, 2006, p. 235 (voir aussi Culy, 1994, p. 1056).
} 
en (1a), ne se vérifie pas. En revanche, ce que les exemples (1a) et (2) ont en commun, c'est que le statut du référent de hän en tant que locuteur ou interlocuteur se définit non pas dans l'espace énonciatif qui engage le locuteur-rapporteur, mais dans celui de la situation rapportée. Il s'agit là d'une différence cruciale entre le logophorique hän et l'antilogophorique se : ce dernier construit la référence à la troisième personne dans l'espace énonciatif du locuteur effectif, que nous notons $\mathrm{Sit}_{0}$ (voir la forme sujet des verbes introducteurs en $1 \mathrm{a}$ et 2 ). L'accès au référent

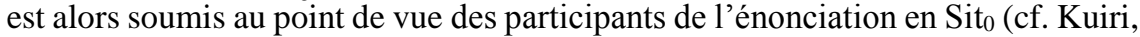
1984, p. 123-124). En tant qu' «objet du discours», le référent de se est une «nonpersonne» (Benveniste, 1966, p. 228-230), dépourvue de statut énonciatif. Le propre de hän, quant à lui, est de situer son référent dans un espace énonciatif

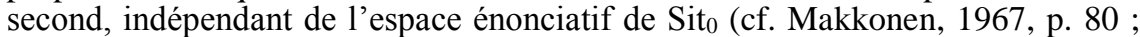
Kallio, 1979 , p. 70), ce qui se voit dans l'emploi des temps verbaux et des expressions déictiques (voir note 6).

Dans les exemples (1a) et (2), hän est utilisé pour désigner un participant saillant du procès mis en mots dans le discours rapporté. La mise en place d'un espace énonciatif distinct de celui de $\mathrm{Sit}_{0}$ permet à hän de faire ressortir la qualité de son référent en tant qu'être intentionnel (cf. Laitinen, 2005, p. 91) : celui-ci se conçoit comme un sujet de conscience (Banfield, 1979 ; Zribi-Hertz, 1989, p. 704-705) du point de vue duquel le procès est présenté (Hagège, 1974, p. 303 ; Laitinen, 2005, p. 93).

Le décrochage par rapport à l'espace énonciatif de $\mathrm{Sit}_{0}$ et la focalisation sur le point de vue du référent de hän peuvent être interprétés comme la non-prise en charge par le locuteur-rapporteur du contenu du discours rapporté. Lorsque le contexte permet d'interpréter hän comme coréférent avec le sujet d'un verbe introducteur tel que sanoa «dire» (ex. 1a), la responsabilité énonciative quant au discours rapporté semble incomber au référent de hän, doté du statut de locuteur secondaire (cf. Hagège, 1974, p. 287 ; Stirling, 1994, p. 219). Mais comme on l'a vu, ce type de coréférence ne s'établit pas nécessairement; elle est résultat d'un calcul référentiel. La capacité indicielle de hän, quel que soit le statut de son référent dans l'espace énonciatif second, réside dans le fait d'associer l'énoncé à une origo ${ }^{8}$ énonciative virtuelle, située à l'extérieur de l'espace intersubjectif de $\mathrm{Sit}_{0}$ qui engage le locuteur et son (ses) interlocuteur(s).

Pour éclairer ce que nous entendons par ce repère virtuel, considérons deux exemples où hän s'emploie dans des énoncés qui ne sont pas des paroles rapportées. En (3), tiré de notre corpus littéraire, il s'agit d'un discours intérieur de la protagoniste (Marja $=\mathrm{A}$ ) dans lequel elle passe en revue des événements qui viennent de se produire : son mari qu'elle a quitté pour s'enfuir avec un autre homme a fait une apparition dans la propriété de ce dernier :

\footnotetext{
${ }^{8}$ Le terme origo est emprunté aux travaux portant sur la deixis. Selon Hanks (1992, p. 50), le sens indiciel d'une expression déictique détermine l'origo à laquelle se rattache la relation qu'elle spécifie. Nous considérons que le logophorique hän spécifie une relation énonciative et non une relation déictique, cette dernière étant liée à la localisation du référent dans l'espace cognitif et social du locuteur et/ou de son interlocuteur.
} 
(3)

1 Marja oli vaipunut penkille, pyörtymäisillään...

2 A : «Marja s'était affaissée sur le banc, prête à s'évanouir...»

$\mathrm{Se}_{\mathrm{i}}$ oli ollut tuossa, oli ovea avannut,

il AUX-PRÉT-3 être-PPA là AUX-PRÉT-3 porte-PAR ouvrir-PPA

«Il $\mathrm{l}_{\mathrm{i}}$ avait été là, avait ouvert la porte»

3

oli katsonut tänne sisään.

AUX-PRÉT-3 regarder-PPA ici intérieur-ILL

«et regardé à l'intérieur.»

$4 \quad S e_{\mathrm{i}}$ oli $\quad$ kuullut lapsen huudon, $\mathrm{se}_{\mathrm{i}}$ oli sitä

il AUX-PRÉT-3 entendre-PPA enfant-GÉN cri-GÉN il AUX-PRÉT-3 le-PAR

«Il ${ }_{\mathrm{i}}$ avait entendu le cri de l'enfant et, pour le»

5 rauhoittaakseen painanut oven takaisin kiinni calmer-INF-TRA-POS.3 pousser-PPA porte-GÉN ADV fermé «calmer, ili avait refermé la porte »

6 eikä tullut sisään... Mitä hän $n_{\mathrm{i}}$ on täällä? NÉG-3-ADD venir-PPA intérieur-ILL quoi-PAR LOG être-3 ici «et n'était pas entré... Pourquoi est-ili là ?»

$7 \quad$ Minuako kuulustelemassa? Minuako etsimässä? me-PAR-Q questionner-NMLS-INE me-PAR-Q chercher-NMLS-INE «Pour s'enquérir de moi, me chercher ?» (Aho, Juha, 1911, XII)

Les énoncés qui relatent des états de choses situés dans le passé (lignes 2-6) construisent la référence au mari de Marja à l'aide de l'antilogophorique se «il». Il s'agit de l'interprétation de A sur ce qui s'est passé. La forme de reprise pronominale change à la ligne 6 dans un énoncé interrogatif de type «pourquoi», avec le verbe au présent. Grâce à hän, cet énoncé produit l'effet d'une interrogation qui s'impose soudainement à la conscience de la locutrice. Le logophorique hän dissocie le référent du point de vue de A pour mettre en scène un sujet de conscience dont l'implication dans le procès n'est pas validable dans l'espace énonciatif de $\mathrm{Sit}_{0}$. Plutôt que de mettre en attente une réponse, la forme interrogative indique une valeur d'indétermination, dénuée de prise de position de la part de A en ce qui concerne l'existence ou non d'une bonne raison. Notons en particulier qu'une pondération sur la valeur négative («Pourquoi est-il là ?» $\rightarrow$ «Il ne devrait pas être là», cf. Culioli, 1999, p. 68-69), possible avec le pronom antilogophorique se, est bloquée ici. Ainsi, le recours au logophorique peut être vu comme un moyen d'écarter le point de vue subjectif de A dans la mise en mots de l'état de choses auquel elle est confrontée'.

Dans l'état de choses mis en mots par l'énoncé Mitä hän on täällä? «Pourquoi est-il $1_{\text {LOG }}$ là?», le référent de hän n'a pas de rôle énonciatif. D'un autre côté, hän n'instaure pas non plus de rapport d'altérité entre la locutrice et l'objet de son discours, ce qui est caractéristique de l'emploi de l'antilogophorique se. La référence construite à l'aide de hän est désembrayée de Sit $_{0}$ (cf. Philippe, 1995) : elle permet de dépasser non seulement l'opposition personne/non-personne, mais aussi celle entre les différentes positions subjectives qui peuvent être prises dans

9 Pour le dire autrement, il s'agit, pour A, de retarder le moment de faire face au fait que son mari soit là pour la chercher. 
l'espace intersubjectif de $\mathrm{Sit}_{0}$. Le repère virtuel qui constitue l'origo de la référence par hän est à la fois non spécifié et absolu : c'est la position que peut assumer tout valideur virtuel. Nous notons ce repère $S_{X}{ }^{10}$.

La mise en place d'un espace énonciatif second avec une origo énonciative virtuelle peut être liée aussi au refus du locuteur de se prononcer sur un état de choses, que ce soit par ignorance, indifférence ou pour une autre raison. Dans ce cas, le référent de hän n'est pas forcément un être intentionnel :

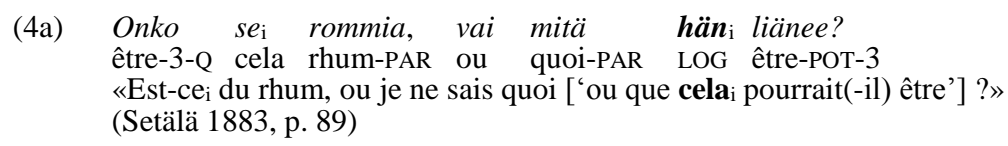

En (4a), il s'agit de catégoriser un référent inanimé, désigné dans un premier temps à l'aide de l'antilogophorique se «cela». La première suggestion d'attribut (rommia «du rhum»), soumise à la modalité interrogative du verbe, est suivie d'une séquence introduite par le coordonnant vai «ou», qui renonce à la catégorisation («ou je ne sais quoi»). Dans cette séquence, le logophorique hän est accompagné du verbe au mode potentiel et de l'élément mitä «quoi» indiquant la non-sélection d'une valeur particulière pour l'attribut. Le mode potentiel sert à envisager l'état de choses $\mathbf{Z}$ («appartenance du référent de hän à une catégorie») comme une possibilité théorique qui n'a pas d'ancrage dans un système temporel (cf. Forsberg, 1998, p. 304 ; 2000, p. 13-14 ; Leech, 1987[1971], p. 113-116 ; Peltola, 2011, p. 38-40). La valeur virtualisante de ce mode contribue ici à indiquer l'inaccessibilité, pour le locuteur, de l'espace énonciatif second dans lequel hän inscrit son référent ${ }^{11}$.

Le marqueur discursif - $h A n$ diffère du pronom logophorique hän en ce qu'il ne réfère pas. On peut supposer que le changement de catégorie a commencé dans des contextes syntactico-discursifs où le recours à hän n'était pas motivé par sa capacité référentielle, mais par sa logophoricité ${ }^{12}$. La perte de référentialité a été

10 On s'inspire ici de la notion de repère fictif $S_{X}$ qui a été appliquée à l'analyse de la particule ved' en russe par Paillard \& Markowicz (1986) et que l'on trouve chez De Vogüé (1987, p. 121-122) examinant les séquences en si en français ; voir aussi De Vogüé \& Paillard (1987, p. 28-31) qui définissent le repère $S_{X}$ comme servant de «représentant à l'hétérogénéité première, c.à.d. non construite».

Notons par ailleurs que du fait du désembrayage de Sito, la référence construite par hän est apte à produire des effets discursifs d'identification, notamment l'effet d'un locuteur qui s'identifie au point de vue du référent de hän (voir Seppänen, 1998, p. 83-92 ; Laitinen, 2005, p. 92-93). En outre, dans la narration à la troisième personne, hän peut être utilisé pour désigner le protagoniste au point de vue duquel le narrateur s'identifie, l'antilogophorique se servant à désigner les référents soumis au point de vue du protagoniste.

${ }_{11}$ Cf. le mode subjonctif qui, dans certaines langues, est utilisé dans les énoncés relevant du discours rapporté (Stirling, 1994, p. 219); pour les cooccurrences de hän et du mode potentiel, voir Forsberg, 1998, p. 294-296.

12 Pour ce qui concerne l'âge de la particule, ses premières attestations écrites datent du XVIII ${ }^{\mathrm{e}}$ siècle (Forsman Svensson, 2003, p. 52, 73-74). Selon Laitinen (2004), l'expansion 
accompagnée de la perte d'autonomie en tant que mot : - $h A n$ est un enclitique qui s'adapte au vocalisme de sa base. L'enclitisation a concerné les formes sujets hän, qui sont monosyllabiques, auxquelles a été antéposé un autre constituant phrastique. En effet, le marqueur discursif $-h A n$ a une position fixe dans l'agencement linéaire de l'énoncé : il est toujours suffixé au premier constituant ${ }^{13}$. Ce constituant en tête d'énoncé est susceptible de constituer un foyer de contraste (Vilkuna, 1989, p. 84-120), ce qui entraîne des ordres des constituants de type $\mathrm{C}_{-h A n} \mathrm{~S}(\mathrm{C}) \mathrm{V}, \mathrm{V}_{1-h A n} \mathrm{~S}(\mathrm{C})\left(\mathrm{V}_{2}\right)(\mathrm{C})$, etc. ${ }^{14}$

Un des contextes propices à l'enclitisation de $h A n$ est constitué par des énoncés à la forme interrogative, avec l'élément interrogatif en position initiale (Laitinen, 2002). Tel est le cas dans l'exemple (4a) ci-dessus, où hän pourrait être analysé non seulement comme un pronom sujet, mais aussi comme une particule sans référence, suffixée au mot interrogatif $(4 b)$ :

$$
\begin{array}{llllll}
\text { Onko } & \text { se } & \text { rommia, } & \text { vai } & \text { mitä-hän } & \text { liänee? } \\
\text { être-3-Q } & \text { cela } & \text { rhum-PAR } & \text { ou } & \text { quoi-PAR-hAn } & \text { être-POT-3 }
\end{array}
$$
«Est-ce du rhum, ou je ne sais quoi ?»

La réanalyse de hän comme non référentiel est structurellement fondée sur la possibilité d'avoir, dans les unités complexes, des sujets «zéros» anaphoriques (voir Hakulinen \& Laitinen, 2008, p. 168-169). En (4b), le changement de statut catégoriel de hän ne semble pas agir sur l'interprétation de l'énoncé. Les motivations pour la réanalyse apparaissent plus nettement en comparant (4a) à l'exemple (5a) où le verbe accompagné d'une occurrence de $h A n$ potentiellement ambiguë met en scène un référent humain et se situe dans un contexte dialogal.

Aux lignes 5-6 de l'extrait en (5a) se trouve un couple question-réponse dans lequel $\mathrm{B}$ refuse d'apporter la réponse attendue à la question de $\mathrm{A}$ (pour des données dialectales du même type, voir Latvala, 1899, p. 55) :

(5a) ((Après avoir disparu pendant un an, Marja (B) est revenue chez Juha (A), son mari. Dans le dialogue ci-dessous, celui-ci évoque son départ, pensant qu'elle avait été enlevée, alors qu'en réalité, Marja est partie de son plein gré. Aux lignes 1 et 5, l'antilogophorique se «il» réfère à Shemeikka, le supposé ravisseur russe.))

de l'emploi particulaire de $h A n$ était en cours au XIX ${ }^{\mathrm{e}}$ où a commencé la standardisation de la langue. À la différence du pronom hän (voir note 2 ), l'enclitique - $h A n$ n'a pas suscité l'intérêt des réformateurs de langue et a pu s'introduire dans le finnois standard sans être soumis à des mesures prescriptives. Le corpus littéraire que nous analysons ici reflète une situation linguistique non standardisée, notamment en ce qui concerne l'emploi des pronoms de troisième personne, qui ressemble à celles attestées par les études descriptives de différents dialectes du finnois publiées à la fin du XIX ${ }^{\mathrm{e}}$ siècle (par ex. Lönnbohm, 1879; Sirelius, 1894 ; Latvala, 1895 ; 1899 ; pour la langue de J. Aho, voir Laitinen, 2011).

${ }_{13}$ Cette position est connue dans la littérature comme «la position de Wackernagel» (pour le finnois, voir Laiho, 1984, p. 42).

14 Pour les cas similaires en russe, où le point d'incidence de l'enclitique est à la fois le premier mot de la portée et un foyer de contraste, Bonnot (2002) parle de double orientation, initiale et focale. 
1 A : - Pitikö se sinua siellä hyvinkin pahasti?

«-T'a-t-ili beaucoup maltraitée là-bas ?»

2 Nyt se alkaa sitä kysellä. Hän oli jo luullut, ett'ei Juha siihen puutu. Mitä minä sille sanon?

«Le voilà qui commence à m'interroger. Elle croyait déjà que Juha ne s'inquiéterait plus de cela. Que vais-je lui dire ?»

4 B : - Missä siellä? Niin, sielläkö?

«-Où ? Ah, oui, là-bas ?»

$5 \mathrm{~A}:-M i t e n k \ddot{a} \quad s e_{\mathrm{i}}$ sai sinut venheeseen?

comment il réussir à prendre-PRÉT-3 toi-ACC barque-ILL

«-Comment a-t-ili réussi à te prendre dans sa barque ?»

6 B : -Miten-hän lie saanut.

comment-hAn AUX-POT réussir à prendre-PPA

«-Ça, comment il a pu réussir ?...»

7 A : - Menitkö tainnoksiin, koska et nostanut päätäsikään? etkä saanut jäsentäkään

8 liikautetuksi?

«- Tu avais perdu connaissance, puisque tu n'as même pas levé la tête et que tu ne pouvais pas non plus remuer un seul membre?»

9 B : - Enkö saanut?

«- Vraiment ?» (Aho, Juha, 1911, XV)

(5b) - Miten hän $n_{\mathrm{i}}$ lie saanut.

comment LOG AUX-POT-3 réussir à prendre-PPA

«- Ça, comment $\mathbf{i l}_{\mathrm{i}}$ a pu réussir ?...»

L'énoncé en - hAn (ligne 6) fonctionne comme réponse à une interrogation partielle de type miten «comment». Le verbe de la question (saada + cas illatif «réussir à prendre quelque part») est au prétérit de l'indicatif et son sujet est réalisé par l'antilogophorique se «il», c'est-à-dire que le locuteur A assume la réalité de l'état de choses $\mathbf{Z}$, «il a réussi à te prendre dans sa barque», qu'il énonce.

L'énoncé-réponse de la locutrice B reprend le verbe de la question, mais en modifie les indices aspectuo-temporels et modaux : le prétérit de l'indicatif (sai) est remplacé par le potentiel du passé (lie saanut). Comme en (4a), il s'agit d'exprimer une possibilité théorique : le procès est pris en considération sans qu'il soit ancré dans le système temporel construit en relation avec $\mathrm{Sit}_{0}$, mais cette-fois il est envisagé sous l'aspect accompli. Ainsi, l'état de choses $\mathbf{Z}$, initialement mis en mots par A, n'est ni validé ni invalidé, mais évoqué comme une idée. L'énoncéréponse répète aussi le mot interrogatif miten $(k \ddot{a})^{15}$ «comment» auquel est ajouté l'élément $-h A n$ qui se substitue à l'antilogophorique $s e$ «il» de la question.

Par sa réponse, B exprime une prise de distance par rapport à l'état de choses $\mathbf{Z}$. Le procédé de distanciation, qui repose sur le mode potentiel et l'élément $-h A n$, crée un effet d'indifférence qui est interprété par $\mathrm{A}$ comme l'incapacité de $\mathrm{B}$ à

${ }^{15}$ Le suffixe -kä qui disparaît à la ligne 6 est un élément entrant dans la formation de beaucoup de mots outils de la grammaire finnoise : il est, en règle générale, présent dans les formes de deux syllabes (mi-kä «que/quoi.NOM») et optionnel dans les formes comportant plus de deux syllabes. Aucune analyse sémantique de cet élément n'existe à notre connaissance. 
répondre à sa question (voir lignes $7-8$ ) ; du point de vue de $\mathrm{B}$, la distanciation correspond à sa réticence à répondre (voir lignes 2-3).

Si l'élément - $h A n$ se présente dans le texte écrit comme une particule enclitique, l'agencement syntaxique de l'énoncé permettrait de l'analyser aussi comme le pronom sujet (5b). Cependant, la question de l'appartenance catégorielle de $h A n$ n'est pas anodine ici. En tant que pronom, hän se rapporterait au participant agent $\mathrm{du}$ procès. Son référent serait susceptible d'être interprété comme un sujet de conscience dont le point de vue sur le procès n'est pas validable dans l'espace intersubjectif de $\mathrm{Sit}_{0}$ (cf. ex. 3 ci-dessus). Dans l'interprétation de hän comme particule non référentielle, ce qui disparait, c'est la focalisation sur le point de vue d'un sujet de conscience.

Les exemples (1a), (2) et (3) auront permis de voir que la définition du statut du référent de hän dans l'espace énonciatif second (locuteur, interlocuteur, sujet de conscience sans rôle énonciatif) dépend du contexte plus large ${ }^{16}$. D'un autre côté, on a vu que les effets interprétatifs particuliers se neutralisent en présence de certains éléments dans l'énoncé, notamment un élément interrogatif et le mode potentiel. Ces énoncés marqués par l'indétermination d'une partie des repérages ont ceci de particulier que l'animacité du référent de hän - condition pour sa conception comme sujet de conscience - n'est pas un trait nécessaire (ex. 4a). Enfin, le contexte ne motivant plus l'interprétation du référent comme un sujet de conscience, toute la référentialité de hän devient caduque. Cela concerne en particulier la position sujet où la référence peut être maintenue par des zéros anaphoriques.

En tant que marqueur discursif, - $h A n$ a une portée qui s'étend sur la relation prédicative de l'énoncé. En ajoutant $-h A n$ dans son énoncé, le locuteur indique qu'il n'est pas l'instigateur de la prédication exprimée : l'établissement de celleci s'associe au repère virtuel $S_{X}$. En revanche, le mode sous lequel la prédication est mentionnée dans le discours relève du point de vue du locuteur sur le procès. Il s'agit alors, non pas d'un véritable «discours dans le discours», avec les repérages temporels et modaux indépendants de ceux du discours premier, comme cela peut être le cas avec l'emploi pronominal de hän, mais d'un double repérage de l'énoncé dans la scène énonciative, schématisé par la figure 1 :

16 Pour un examen plus détaillé des emplois du logophorique hän, voir Laitinen, 2005. 
(relation prédicative validée)

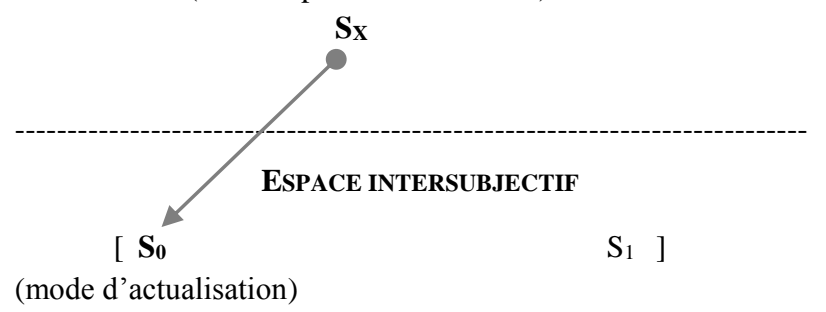

Figure 1 : Double repérage énonciatif de l'énoncé en - $h A n$.

L'espace intersubjectif de la scène énonciative est organisé par des positions subjectives $S_{0}$ et $S_{1}$ auxquelles un énoncé peut être associé de différentes façons (voir Paillard, 2009) ${ }^{17}$. Le mode d'actualisation de la prédication exprimée par l'énoncé en - $h A n$ (le mode potentiel en 5a) se détermine depuis la position subjective $\mathrm{S}_{0}$. Cette position se trouve dans un rapport d'altérité avec la position $S_{1}$. Cette dernière, qui est seconde par rapport à $S_{0}$, est virtuellement une position correspondant à une mise en cause de la position $S_{0}$ (Paillard, 2009). Il s'agit notamment de la position d'un interlocuteur qui se sépare de la position $\mathrm{S}_{0}$.

Les énoncés en - $h A n$ organisent l'espace intersubjectif de sorte que la position $\mathrm{S}_{1}$ soit marginalisée. On peut observer l'effet de cette marginalisation dans l'exemple (5a) ci-dessus, où l'énoncé en $-h A n$ reprend une relation prédicative mise en mots par l'interlocuteur A. Ce qui est attendu dans le contexte, c'est la validation de la prédication par B. Or, dans la reprise, la responsabilité énonciative quant à la prédication est déplacée vers le repère virtuel $S_{X}$. Située à l'extérieur de l'espace intersubjectif, cette position décrochée est hors de portée de la confrontation des positions subjectives $S_{0}$ et $S_{1}$. Ainsi, l'énoncé en - $h A n$ indique que pour $\mathrm{B}$, les déterminations de la relation prédicative, non seulement sa validation ou non, qui est laissée en suspens grâce au mode potentiel, mais aussi la sélection d'une valeur spécifique pour le complément de type «comment», ne constituent pas un enjeu intersubjectif.

Le rattachement de l'énoncé au repère $S_{X}$ peut être considéré comme un procédé permettant le dépassement d'un conflit intersubjectif. En effet, les contextes d'emploi des énoncés en - $h A n$ se caractérisent par un conflit des points de vue dans l'espace intersubjectif. C'est le cas aussi en (5a) où $\mathrm{A}$ croit qu'un étranger lui a ravi sa femme, alors que B sait qu'elle est partie de son plein gré. À l'aide de l'énoncé en $-h A n, \mathrm{~B}$ évite aussi bien la validation de la croyance de A que l'introduction dans le discours d'un savoir dont elle dispose et qui contraste avec les attentes de A.

17 Pour une troisième position «intermédiaire», $S^{\prime}{ }_{0}$, voir Paillard, 2009, p. 116-117. 
Pour récapituler, nous avons tenté de démontrer que tout en perdant sa référentialité, $-h A n$ a conservé sa capacité à mettre en place un repère énonciatif situé à l'extérieur de l'espace énonciatif de $\mathrm{Sit}_{0}$. La définition de la fonction du marqueur discursif - $h A n$ a été formulée en termes de double repérage de l'énoncé : l'établissement de la relation prédicative est associé au repère virtuel $S_{X}$, le locuteur ne prenant en charge que le mode, quel qu'il soit, sous lequel la relation prédicative est actualisée dans le discours. De ce fait, le marqueur discursif $-h A n$ est un moyen pour le locuteur de prendre en considération, donc «prendre au sérieux» (cf. Filippe-Deswelle, 2014), une relation prédicative dont il n'est pas l'instigateur et dont les déterminations (la validation ou non) ne constituent pas, pour lui, un enjeu intersubjectif.

\section{LA DÉSASSERTION}

Les énoncés à la forme interrogative ne sont pas le seul foyer possible pour la réanalyse de $h A n$. Nous analyserons dans cette partie trois exemples d'énoncés commençant par une forme verbale. Comme dans l'exemple (5a), il s'agit de reprendre une relation prédicative déjà construite dans le discours en la soumettant à une modalité verbale dont l'expression se situe en tête d'énoncé. D'une part, nous nous pencherons ici sur la question de la valeur désassertive que - $h A n$ donne aux énoncés non interrogatifs. D'autre part, on verra comment $h A n$, après avoir perdu son interprétation référentielle, s'est défait de son statut syntaxique en tant que marque de la troisième personne pour étendre son emploi auprès des verbes à la première et à la deuxième personne.

\subsection{Modalité déontique de permission}

Dans l'exemple (6a), l'énoncé en - $h A n$ se situe dans le prolongement d'un passage narratif qui constitue le début de la réponse à une question d'opinion (ligne 4). Dans les énoncés narratifs, le protagoniste est d'abord désigné à l'aide d'un nom (ligne 5 : isänsä «son père»), puis les verbes à la troisième personne s'emploient sans pronom sujet (lignes 7-8). À la différence des formes pronominales $s e_{\mathrm{ANTIOG}}$ et $h \ddot{a} n_{\mathrm{LOG}}$, le «zéro» anaphorique ne spécifie pas son origo énonciative, qui se détermine au cas par cas en fonction du statut de l'énoncé dans la scène énonciative. Ici, le zéro anaphorique des énoncés narratifs (lignes 7-8) s'inscrit dans la continuité de la référence pronominale antilogophoprique de la ligne 2 (niillä «ils-ADE/leur» désignant les Shemeikka dont le protagoniste fait partie). D'un autre côté, ne pas utiliser un pronom sujet peut fonctionner comme indice d'une attitude affective du locuteur (Hakulinen \& Laitinen, 2008), telle que l'empathie envers le protagoniste :

(6a) ((A est une femme abandonnée par son amant, Shemeikka ; B est la mère de celui-ci.))

$1 \mathrm{~B}: \quad-(\ldots)$ Shemeikat eivät välitä vanhoista, Shemeikka-PL NÉG-3.PL se soucier vieux-PL-ÉLA $\ll-(\ldots)$ les Shemeikka ne se soucient pas des vieilles,» 


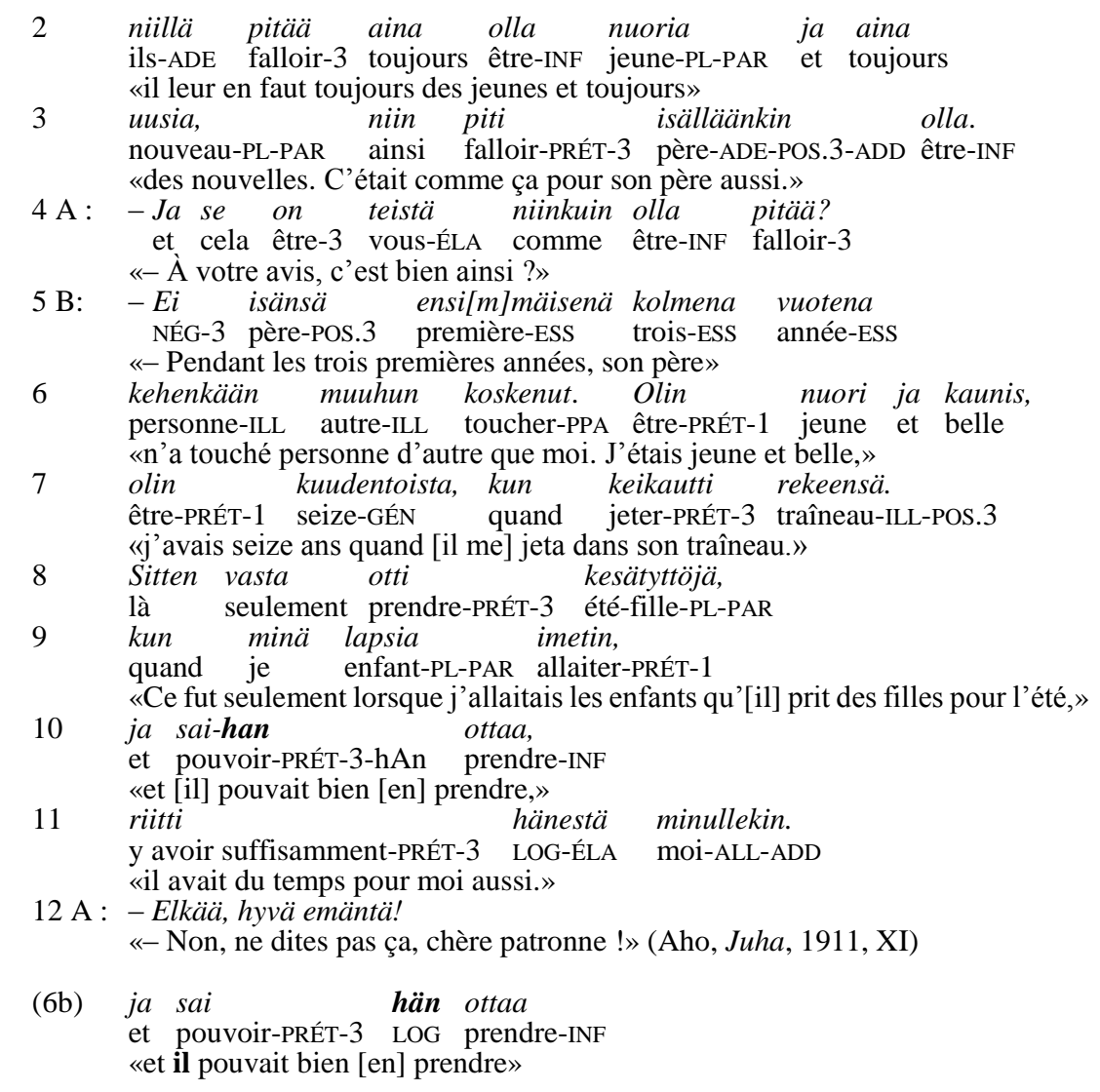

L'énoncé en - $h A n$ (ligne 10), qui est introduit par le coordonnant ja «et», s'interprète comme une prise de recul de la locutrice $\mathrm{B}$ sur l'état de choses $\mathbf{Z}$ «il prit des filles pour l'été», mis en mots dans le passage narratif (ligne 8). B reprend le verbe ottaa «prendre » pour le soumettre à la modalité déontique de permission. L'apport de l'énoncé se réduisant à cette modalité, le verbe modal saada «pouvoir» est situé en position initiale. Ce dernier est porteur de l'enclitique - $h A n$, qui présente une ambiguïté catégorielle du même type qu'en (5a) : il est orthographié comme une particule enclitique, mais pourrait, en principe, avoir aussi la fonction de pronom sujet (6b).

Dans la reprise du verbe ottaa «prendre», la responsabilité énonciative quant à la relation prédicative (mettant en mots l'état de choses $\mathbf{Z}$ ) est associée, à l'aide de $-h A n$, au repère virtuel $S_{X}$. Le décrochement par rapport à $S_{i t}$ signifie que pour $\mathrm{B}$, l'établissement de cette relation prédicative, plus précisément la prédication 
d'existence de $\mathbf{Z}$, ne constitue pas en soi un enjeu intersubjectif. La prédication associée au repère $S_{X}$ est le support de la modalité de permission qui, quant à elle, relève du point de vue de $\mathrm{B}$ en tant qu'engagée dans l'espace intersubjectif de $\mathrm{Sit}_{0}$ : il s'agit, pour B, d'exprimer son ajustement à l'état de choses $\mathbf{Z}$.

L'énoncé en - $h A n$ produit un effet de dédramatisation auquel contribue aussi l'énoncé suivant qui apporte une justification à la modalité de permission (ligne 11 : riitti hänestä minullekin «il avait du temps pour moi aussi»). En effet, l'approbation de $\mathbf{Z}$ par B contraste avec les attentes de l'interlocutrice A (voir lignes 4 et 12). Le recours à $-h A n$ pour indiquer que $\mathbf{Z}$ est pris en considération en tant qu'existant indépendamment des positions subjectives $\left(S_{0}\right.$ et $\left.S_{1}\right)$ que $A$ et $B$ pourraient prendre en $\mathrm{Sit}_{0}$ est alors un moyen pour B de ne pas entrer en conflit ouvert avec $\mathrm{A}$.

L'ambiguïté catégorielle de $-h A n$ mérite encore un commentaire. $\mathrm{Si}$ on interprétait $h A n$ comme référentiel, mettant en scène un sujet de conscience, cela donnerait la possibilité de considérer que la modalité permissive de l'énoncé reflète le point de vue du référent de hän, et non pas celui de B. Cette interprétation est écartée en (6a) par le fait que $h A n$ se présente sous forme d'enclitique (-han) qui se distingue du pronom (hän) par son vocalisme. Cependant, l'origine pronominale de la particule $-h A n$ reste bien tangible. Un phénomène symptomatique des foyers de réanalyse de hän est la présence dans le contexte proche d'une occurrence non clitisée de hän, comme ici la forme hänestä, munie d'un suffixe casuel, dans l'énoncé à valeur justificative (ligne 11) ${ }^{18}$.

\subsection{Modalité dynamique}

En (7a), l'énoncé en - $h A n$ se trouve dans un contexte où le point de vue initial de la locutrice sur la situation en cours contraste avec celui de son interlocuteur : Marja (B) a invité Shemeikka (A) à prendre la chope de lait qu'elle a remplie (ligne 3) alors que lui s'attend à ce qu'elle lui donne la chope dans les mains (lignes 710) :

(7a) ((Shemeikka, un Carélien russe, a passé la nuit chez Juha et Marja. Le lendemain matin, il s'approche de Marja qui est en train de passer le lait.))

1 Shemeikka jäi seisomaan, seuraillen Marjaa silmillään, huulilla hymy. Kun

2 Marja oli maidon siivilöinyt, nouti hän pienen tuopin, täytti sen

«Shemeikka se tenait debout, et le sourire aux lèvres suivait Marja du regard. Après avoir passé le lait, la jeune femme alla chercher une petite chope, la remplit»

3 ja pyysi vieraan ottamaan.

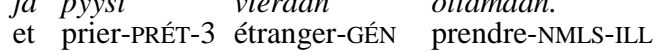

«et pria l'étranger de la prendre.»

18 Les énoncés à valeur justificative sont un des contextes d'emploi courants du logophorique hän (voir Laitinen, 2005, p. 90). 
$4 \quad$ Shemeikka vaan hymyili. Marja kysyi, hymähtäen hänkin, pitikö vieras köyhän talon tarjousta halpana, koska ei kelvannut?

«Shemeikka sourit seulement, et Marja en souriant aussi demanda si l'étranger méprisait la nourriture d'une pauvre ferme puisqu'il n'y goûtait pas.»

$6 \mathrm{~A}$ : - Et taaskaan, emäntä, tunne Karjalan tapoja.

NÉG-2 encore-ADD patronne connaître Carélie-GÉN coutume-PL-PAR

«- Encore une fois, patronne, tu ne connais pas les coutumes de la Carélie.»

7 Meillä ei vieras milloinkaan ota itse,

nous-ADE NÉG-3 étranger jamais prendre lui-même

«Chez nous, un étranger ne prend jamais rien lui-même.»

emäntä hänen käteensä kiidättäa

maîtresse de maison lui-GÉN main-ILL-POS.3 faire arriver vite-3

«La maîtresse de maison veille à ce qu'on lui mette dans les mains»

kaiken mitä tarjoo. Tuulispäänä

tout-GÉN ce que-PAR offrir-3 coup de vent-ESS

«tout ce qu'elle offre. C'est en coup de vent»

10 jo oven suuhun vastaan lennätetään

aussitôt porte-GÉN embouchure-ILL vers faire parvenir rapidement-PAS «qu'on lui fait parvenir les plats aussitôt qu'il se montre à la porte.»

$11 \mathrm{~B}$ : - Saa-han tämän lennättää! pouvoir-3-hAn celle-ci-GÉN faire parvenir rapidement-INF «-Après tout, on peut [te] la faire parvenir!»

12 ja Marja tempasi pöydältä haarikan ja vei sen Shemeikalle (...) «et d'un mouvement brusque, Marja prit la chope en bois sur la table et l'apporta à Shemeikka (...)» (Aho, Juha, 1911, III)

L'énoncé en - $h A n$ (ligne 11) reprend le verbe lennättää «faire parvenir rapidement», utilisé par A dans le tour de parole précédent (ligne 10). Dans la reprise, le verbe est accompagné du modal saada «pouvoir» qui exprime ici la modalité dynamique («possibilité physique»). En même temps, les coordonnées spatio-temporelles changent. À la ligne 10, la forme dite «passive», qui implique un agent humain non spécifié (lennätetään «on fait parvenir rapidement»), décrit les mœurs de la Carélie russe, alors que l'énoncé en $-h A n$, avec son verbe à la troisième personne du singulier et le sujet zéro non spécifié, s'applique à la situation en cours, le pronom objet tämän «celle-ci/la» référant à la chope de lait remplie par Marja.

Les énoncés à sujet zéro non spécifié constituent un environnement syntaxiquement et discursivement intéressant du point de vue de l'expansion de l'emploi particulaire de $-h A n$. Premièrement, ces énoncés qui comportent un verbe à la troisième personne non accompagné de forme sujet offrent un site phrastique où $h A n$, doté d'un statut catégoriel fluctuant, a pu s'introduire aisément grâce à son identité formelle en tant que marque de la troisième personne. Deuxièmement, une certaine convergence sémantique peut être observée entre l'élément $h A n$ et le sujet zéro. Les constructions à sujet zéro se caractérisent par la présence d'une expression modale de possibilité ou de nécessité, ou d'un repérage conditionnel (de type jos... niin... «si... alors...»), c'est-à-dire qu'elles s'emploient pour exprimer des états de choses dont la réalité n'est pas et ne peut pas être validée dans l'espace énonciatif de $\mathrm{Sit}_{0}$. Le «participant zéro» étant non seulement non spécifié, mais aussi référentiellement non spécifique, la réalisation du procès n'est 
envisageable qu'au sein d'un espace énonciatif second avec un valideur virtuel ${ }^{19}$. Le décrochement de l'énoncé de $S_{i} t_{0}$ qu'opère $h A n$ est donc déjà préinscrit dans les constructions à sujet zéro grâce au valideur virtuel qu'elles impliquent. Cette compatibilité non seulement formelle, mais aussi sémantique permet de voir en $h A n$ comme une représentation en filigrane de la «personne zéro», correspondant en (7a) à «quiconque ayant l'intention de faire parvenir la chope», dont l'implication dans le procès dépend du repère virtuel $\mathrm{S}_{\mathrm{X}}$.

D'un autre côté, l'identification au rôle du valideur virtuel que les énoncés à sujet zéro mettent en jeu concerne de manière prioritaire le locuteur et/ou son interlocuteur (Laitinen, 1995). Ainsi, de par leur interprétation situationnelle, les énoncés à sujet zéro sont en concurrence avec les énoncés à sujet explicite de première ou de deuxième personne (voir $7 \mathrm{~b}$ ). On peut alors penser que dans le rapprochement entre formulations concurrentes, les énoncés avec le verbe à la première (ou à la deuxième) personne ont pu emprunter aux énoncés à sujet zéro l'élément $-h A n$ en tant que marqueur discursif dénué de toute référentialité et n'ayant conservé que sa capacité à associer la relation prédicative de l'énoncé au repère virtuel $S_{X}$.

$$
\begin{aligned}
& - \text { Saan-han minä tämän lennättääa } \\
& \text { pouvoir-1-hAn je celle-ci-GÉN faire parvenir rapidement-INF } \\
& \text { «-Après tout, je peux [te] la faire parvenir !» }
\end{aligned}
$$

En (7a), l'énoncé en - $h A n$ produit un effet concessif : si son contenu est différent de la position initiale de $\mathrm{B}$, d'une part, et conforme à la position de l'interlocuteur A, d'autre part, cette dernière n'est pas pour autant adoptée sans réserve. Le marqueur discursif $-h A n$ déplace la responsabilité énonciative concernant la relation prédicative mise en mots par $\mathrm{A}$ à l'extérieur de l'espace intersubjectif. Cela signifie que $B$ ne considère pas que la validation de la prédication $<X$ fait parvenir la chope > constitue un enjeu intersubjectif. En la prenant en considération sous la modalité de possibilité et avec un valideur non spécifié, B se garde la liberté d'agir ou de ne pas agir à sa convenance. Qui plus est, le décrochement de $\mathrm{Sit}_{0}$ permet de donner un ton légèrement ironique à la répétition du verbe lennättää «faire parvenir rapidement», dérivé factitif de lentää «voler (avec des ailes)», dont la fonction est descriptive dans le tour de parole de A (ligne 10).

${ }^{19}$ L'emploi d'un verbe lexical à l'indicatif dans la construction à sujet zéro produit un effet de décrochage de Sito. Un énoncé tel que :

$$
\begin{array}{ll}
\text { Täällä } & \text { ikävystyy. } \\
\text { ici s'ennuyer-3 } \\
\text { «On s'ennuie ici.» }
\end{array}
$$

met en place un espace énonciatif second avec un valideur virtuel au rôle duquel tout valideur peut s'identifier (cf. Laitinen, 1995). De ce fait, la construction à sujet zéro se distingue de la forme verbale «passive» (marquée par le suffixe TA) qui implique également un participant (agent) humain non spécifié, mais qui permet d'exprimer des états de choses ayant un ancrage spatio-temporel déterminé par rapport à Sito (voir ex. $7 \mathrm{a}$, ligne 10) 


\subsection{Modalité négative}

Dans le troisième exemple, l'énoncé en - $h A n$ (ligne 4) s'oppose par sa modalité négative à l'énoncé de l'interlocutrice qui le précède (ligne 1) :

(8a) ((Échange entre Juha (B) et sa mère (A) ; la disparition de la femme de Juha date de presque un an déjà.))

1 A : - Taas se siinä pälyy, sanoi Juhan äiti ylenkatseellisesti rukkinsa takaa. encore il là guetter-3

«- Le voilà encore qui guette, dit d'un air méprisant la mère de Juha derrière son rouet.»

$2 J u h a$, joka taas oli istunut ikkunassa, metsän rantaan tähystämässä, vastasi säpsähtäen:

«Juha qui, de nouveau, était assis près de la fenêtre et scrutait la lisière du bois, répondit en sursautant :»

$4 \mathrm{~B}$ : - En-hän minä mitä päly.

NÉG-1-hAn je rien-PAR guetter

«- Mais je ne guette rien.»

$4 \mathrm{~A}$ : - Vaikka se nyt joskus maailmassa tulisikin sieltä, minne niin mielellään meni... «-Et même si elle ((= la femme de Juha)) revenait un jour de là-bas, où elle est allée avec tant de plaisir...»

6 B : - Liekö tuo mielellään mennyt, eihän sitä tiedä, sanoi Juha säyseästi.

«- Est-elle vraiment partie avec plaisir ? On ne peut pas le savoir, dit Juha tranquillement.» (Aho, Juha, 1911, XIII)

Dans son tour de parole à la ligne 4, B exprime qu'il n'admet pas l'état de choses $\mathbf{Z}$ «Juha guette (quelque chose)» dans lequel il est censé être impliqué. La reprise du verbe pälyä «guetter», utilisé d'abord par A dans la mise en mots de $\mathbf{Z}$ (ligne 1), est accompagnée de l'auxiliaire de négation ${ }^{20}$ situé en tête d'énoncé et porteur de $-h A n$. La négation est renforcée par le pronom indéfini à valeur négative mitä «rien», antéposé au verbe lexical, qui matérialise le complément d'objet, non instancié dans la construction de la ligne 1.

Étant donné que le verbe est à la première personne, la catégorie de - $h A n$ est sans ambiguïté. Il s'agit d'un marqueur discursif qui associe la validation première de la relation prédicative de l'énoncé au repère virtuel $S_{X}$; seule la modalité négative indiquant la non-adhésion de $\mathrm{A}$ à l'état de choses $\mathbf{Z}$ se détermine depuis l'espace intersubjectif.

Le fait de déplacer la responsabilité énonciative quant à la prédication en dehors de l'espace intersubjectif a pour effet d'atténuer le conflit qui oppose a priori le locuteur à son interlocutrice : grâce à $-h A n$, l'énoncé se trouve privé de sa pleine valeur assertive. La valeur assertive est ici entendue comme une question de l'implication du locuteur dans la mise en mots et dans les déterminations de la relation prédicative de son énoncé. Un énoncé assertif $\mathbf{p}$ dit ce que le locuteur, en tant qu'engagé dans l'espace intersubjectif de $\mathrm{Sit}_{0}$, croit ou sait être le cas (ou ne pas être le cas). Du fait de cette implication du locuteur, l'énoncé assertif p s'associe à la position subjective $S_{0}$ de l'espace intersubjectif et en même temps,

20 Il s'agit d'un auxiliaire doté de l'indice personnel. 
il se met en concurrence, virtuellement du moins, avec un énoncé p', correspondant à non-p ou à autre que $\mathbf{p}$, associé à la position $\mathrm{S}_{1}$ (voir figure 1 dans la partie 2).

Le caractère conciliant de l'énoncé négatif en - $h A n$ peut être comparé à l'effet d'objection que produirait, dans un contexte tel que (8a), un énoncé sans $-h A n^{21}$. Il s'agirait d'organiser l'espace intersubjectif avec une altérité forte entre les positions $S_{0}$ et $S_{1}$ (voir $8 b$, ligne 2) : l'énoncé $\mathbf{p}$, associé à la position $S_{0}$, s'interprèterait comme une remise en cause de la position $\mathrm{S}_{1}$ prise par l'interlocutrice dans son énoncé de la ligne 1 (correspondant à $\mathbf{p}$ '). Le marqueur discursif $-h A n$, en revanche, sert à marginaliser la position $S_{1}$ : il permet au locuteur de faire connaître son point de vue sur l'état de choses $\mathbf{Z}$, mis en mots par l'interlocutrice, tout en dépolémisant la mise en mots de $\mathbf{Z}$ : cette dernière n'est pas considérée par B comme un enjeu intersubjectif.

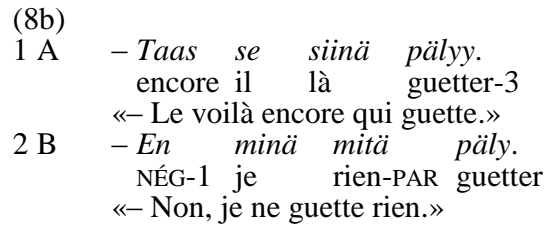

On a vu dans cette partie que les effets de sens que $-h A n$ produit, qui varient d'un exemple à l'autre, dépendent essentiellement de la façon dont l'énoncé répond aux attentes et des liens de celles-ci avec les deux positions subjectives antagonistes $S_{0}$ et $S_{1}$ de l'espace intersubjectif. Au-delà des effets de sens, la propriété commune à tous les exemples réside dans le fait que - $h A n$ prive l'énoncé de sa pleine valeur assertive : il s'agit de désassertions ${ }^{22}$.

Enfin, les exemples (6a), (7a) et (8a) se situent sur un continuum en ce qui concerne l'ambiguïté catégorielle de $-h A n$ et la possibilité de le considérer comme référentiel :

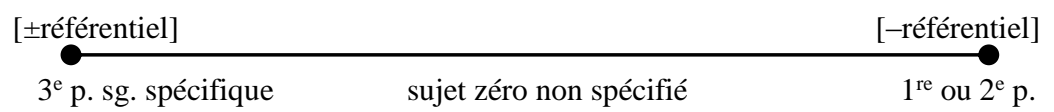

Figure 2. La référentialité de $h A n$ et la personne verbale.

${ }^{21}$ Il est à souligner que l'emploi de $-h A n$ dans l'exemple (8a) trouve une motivation dans la forme de l'énoncé de A (ligne 1), qui utilise l'antilogophorique se «il/le » pour désigner B. De ce fait, l'énoncé de A construit B seulement comme participant impliqué dans le procès, et non pas comme à la fois interlocuteur et participant impliqué, ce que ferait le pronom de deuxième personne (sinä «tu»). Dans son énoncé, $\mathrm{B}$ redéfinit son rôle comme à la fois locuteur et participant impliqué, mais déplace la responsabilité quant à son implication supposée dans le procès à l'extérieur de l'espace intersubjectif.

22 Cette idée, formulée en termes différents, se trouve chez A. Hakulinen (2001b[1976], p. 48-57) qui estime que - $h A n$ sert à modifier la force illocutoire de l'énoncé. 
Ce continuum peut se lire comme une hypothèse sur l'expansion de l'emploi de $-h A n$ en tant que marqueur discursif. Dans le processus où il s'est agi de passer d'une forme sujet de troisième personne du singulier à une particule non référentielle suffixable à un verbe à la première ou à la deuxième personne, un rôle de passerelle a pu être joué par les constructions à sujet zéro qui sont non seulement formellement (verbe à la troisième personne du singulier), mais aussi sémantiquement (implication d'un valideur virtuel relevant du repère $S_{X}$ ) compatibles avec l'élément $-h A n$.

\section{L’ACCESSIBILITÉ DE LA RELATION PRÉDICATIVE}

Les exemples analysés dans les parties précédentes ont en commun le fait que la relation prédicative mentionnée dans l'énoncé en - $h A n$ est préconstruite dans le contexte linguistique précédent. Dans cette partie, nous nous intéresserons à des cas où le statut de préconstruit de la prédication ne provient pas d'une matérialisation antérieure dans le discours.

\subsection{Mirativité}

Les études antérieures ont souvent posé la question de la définition de - $h A n$ en termes de statut informationnel de l'énoncé (L. Hakulinen, 1999[1951], p. 44 ; A. Hakulinen, 2001b[1976] ; Välimaa-Blum, 1987 ; Forsberg 2006, p. 85-87). Selon la définition communément admise (ISK, 2004, § 830), - $h A n$ indique que l'énoncé fait appel à des connaissances partagées des participants de la situation d'énonciation. Cette idée s'applique pourtant difficilement à certains emplois bien répertoriés de $-h A n$, notamment à celui qui consiste à exprimer la surprise ou la prise de conscience soudaine : le locuteur est alors confronté à une information nouvelle.

La prise de conscience peut être déclenchée par la situation physique de l'énonciation ou résulter d'un «déclic» sans stimulus apparent, se produisant dans la tête d'un locuteur préoccupé par un problème quelconque, comme en (9) :

(9)

1 Se oli ensin ollut Juhalle niinkuin tapaturma, niinkuin olisi Marja häneltä

2 joutunut jonnekin, josta ei kuulunut huutoa, josta se ei päässyt omin voimin

3 enemmän kuin elukka suosta tai metsän peitosta.

«D'abord Juha avait eu l'impression qu'il était arrivé un accident ; il lui semblait que Marja était tombée dans un lieu d'où son cri ne pouvait s'entendre et d'où, par ses propres moyens, elle ne parvenait pas plus à sortir qu'un animal ne sort du marécage ou de la forêt où il s'est égaré.»

$4 \quad$ Mutta kun hän istui venheessään ja souti ja souti, alkoi hänelle selvitä

«Mais lorsqu'il fut assis dans sa barque, il rama, rama, et commença à entrevoir»

mitä oikeastaan oli tapahtunut.

quoi-PAR en réalité AUX-PRÉT-3 se produire-PPA

«ce qui était arrivé :» 
6 Marja-han oli ryöstetty,

Marja-hAn AUX-PRÉT-3 enlever-PPA.PAS

«Marja avait été enlevée !»

$7 \quad$ vieras mies oli hänet väkisin vienyt, oli vienyt häneltä vaimon.

«L'étranger l'avait emmenée de force, il lui avait ravi sa femme.»

(Aho, Juha, 1911, IV)

L'énoncé en -hAn (ligne 6) exprime un état de choses $\mathbf{Z}$ qui s'impose à la conscience de Juha, protagoniste-expérient au point de vue duquel le narrateur s'identifie. La relation prédicative n'est pas construite depuis la position $\mathrm{S}_{0}$ : il s'agit, pour le locuteur, de donner une actualisation dans le discours à une prédication «toute faite » (cf. Bonnot, 2004, p. 228-229; Montaut, 2006) dont la préconstruction s'associe, grâce à $-h A n$, au repère virtuel $\mathrm{S}_{\mathrm{X}}$. Ici, le constituant initial porteur de $-h A n$ n'est pas un foyer de contraste, mais l'énoncé dans son ensemble forme un «bloc rhématique» insécable, qui exprime «ce qui était arrivé» (voir ligne 5). On peut noter en particulier qu'aucune pause ne pourrait s'insérer entre le complément (Marja) et le verbe (oli ryöstetty « avait été enlevée), à la différence d'un énoncé s'organisant en support (thème) et en apport (rhème) ${ }^{23}$.

La mirativité ${ }^{24}$ résulte du fait que le contenu de l'énoncé en - $h A n$ contraste avec les attentes du locuteur qui ont leur ancrage dans l'espace intersubjectif de $\mathrm{Sit}_{0}$ et qui sont accompagnées d'incertitudes : en (9), Juha imagine d'abord que la disparition de sa femme était un curieux accident. Le fait que la responsabilité énonciative concernant l'état de choses $\mathbf{Z}$ dont le locuteur prend conscience plus ou moins soudainement soit déplacée en dehors de l'espace intersubjectif a pour effet de le présenter comme une évidence inébranlable depuis les positions subjectives $S_{0}$ et $S_{1}$. Par là même, l'énoncé en - $h A n$ anéantit les attentes antérieures ainsi que les incertitudes qui y sont liées.

\subsection{Les justifications}

La justification de l'énoncé précédent figure parmi les fonctions courantes des énoncés en -hAn. En (10), il s'agit de justifier un énoncé injonctif:

(10) ((Après avoir été meurtri par le départ de sa femme, Juha semble avoir tourné la page.))

1 (...) kun Juha tuli illalla kotiin, oli hän yhäkin niinkuin olisi ollut yhtä hyvillään

2 kuin lähtiessä̈̈n. Hekotteli, naurakehteli, hoilotteli saunassa kylpiessään. «(...) quand Juha, le soir, rentra à la maison, il semblait toujours d'aussi bonne humeur. En prenant son bain dans l'étuve, il riait à gorge déployée et menait grand bruit.»

3 A - Mennä lauvantaina oli minulla tässä vielä eukko löylyä lyömässä,

«- Samedi dernier j'avais encore une femme ici, pour faire de la vapeur,»

${ }^{23}$ Les traits prosodiques de ce type d'énoncés mériteraient une étude à part.

${ }^{24}$ Par mirativité, on entend ici une catégorie sémantique d'énoncés qui expriment une information nouvelle et inattendue pour le locuteur (DeLancey, 2001, p. 369-370.). 
nyt ei olekaan - ei olekaan - ei tulekaan - lallalaa - hilutullallaa!

«il n'y en a plus maintenant, il n'y en a plus. Elle ne reviendra pas, tra la la la la, houp, la la !»

5

Lÿ̈ sinä, äiti, pojallesi löylyä.

faire-IMP.2 toi mère fils-ALL-POS.2 vapeur-PAR

«Fais de la vapeur pour ton fils, mère.»

Sama-han se on, kuka lyö. Häh?

égal-hAn cela être-3 qui faire-3 INTERJ

«Peu importe qui le fait. Hein ?»

7 Meni-meni-meni-meni minulta akka ryssän rekeen! Vaan entäpä menikin? Otetaan uusi sijaan? Mitä? kysyi hän lakaten hutkimasta. Saa kai sen ottaa

uudenkin?

«Elle est partie... partie... partie, ma patronne est partie dans le traîneau d'un Russe. Et puis après ? On en prendra une nouvelle. Est-ce possible ? demandat-il en cessant de se fustiger avec les branches de bouleau, est-ce possible d'en prendre une autre ?» (Aho, Juha, 1911, VII)

L'énoncé injonctif (ligne 5 : Lyö sinä, äiti, pojallesi löylyä. «Fais de la vapeur pour ton fils, mère.») met en mots un état de choses $\mathbf{Z}_{1}$ qui n'est que virtuel. La construction de la relation prédicative et la mise en attente de la valeur positive de cette relation se font par le locuteur depuis la position $\mathrm{S}_{0}$ de l'espace intersubjectif; d'un autre côté, l'énoncé injonctif met en jeu la position $\mathrm{S}_{1}$ qui est le support de deux valeurs non sélectionnées, la validation et la non-valisation de $\mathbf{Z}_{1}$, et qui implique l'interlocutrice en tant que valideur potentiel de $\mathbf{Z}_{1}$ (De Vogüé \& Paillard, 1987, p. 32 ; Paillard, 1992, p. 82-83).

L'état de choses $\mathbf{Z}_{1}$ qui est encore non réalisé contraste avec un état de choses antérieur $\mathbf{Z}_{0}$, «la femme de Juha fait de la vapeur» (voir ligne 3). Proposer un nouvel arrangement implique que Juha n'attache plus d'importance au fait que ce ne soit pas sa femme qui fasse de la vapeur. L'énoncé en - $h A n$ matérialise cette implication (ligne 6 : Samahan se on, kuka lyö. «Peu importe qui le fait»). Sa fonction est donc de faire savoir à l'interlocutrice que contrairement aux attentes antérieures, il n'y a pas d'obstacle à la validation de l'état de choses $\mathbf{Z}_{1}$.

La valeur justificative de l'énoncé en - $h A n$ s'appuie sur le fait que la prédication <peu importe qui le fait> n'est pas construite depuis la position subjective $\mathrm{S}_{0}$, mais elle est associée au repère $S_{x}$. Ainsi, l'indifférenciation du choix de valideur pour l'état de choses $\mathbf{Z}$ «[ ] fait de la vapeur» est présentée comme n'étant pas un enjeu intersubjectif. Outre son rapport justificatif avec l'énoncé injonctif, l'énoncé en $-h A n$ porte une nuance concessive qui résulte de son rapport contrastif avec la position antérieure de Juha. En actualisant dans son discours la prédication <peu importe qui le fait>, le locuteur s'ajuste aux circonstances qui s'imposent à lui.

Les énoncés en - $h A n$ qui fonctionnent comme justifications peuvent aussi faire appel à des connaissances déjà établies, supposées partagées. En (11), il s'agit d'un discours intérieur de Juha dans lequel il se donne des raisons pour abandonner son travail dans la forêt et rentrer à la maison (voir ligne 2) :

(11) ((La servante est venue apporter à manger à Juha qui a travaillé dans la forêt. La narration se fait du point de vue de Juha.))

$1 \quad$ Piika alkoi avata tuomaansa nyyttyä, 
2 «La servante commençait à ouvrir son panier»

mutta Juha sanoi menevänsä kotiin.

mais Juha dire-PRÉT-3 aller-PPR-POS.3 maison-ILL

«quand Juha lui dit qu'il rentrait à la maison.»

3 Jääköön Kaisa tänne vielä kerppuja taittamaan.

«Que Kaisa reste encore un moment pour couper du feuillage,»

$4 \mathrm{Ei}$ tuntunut enää jaksavan hakata.

NÉG-3 se sentir-PPA plus avoir la force de-PPR-GÉN battre-INF

«lui ne se sentait plus la force de continuer son travail,»

5 Ja oli-han lauvantai, ja olivat verkotkin laskettavat.

et être-PRÉT-3-hAn samedi

«et après tout, on était samedi, il fallait aussi tendre les filets.»

(Aho, Juha, 1911, I)

L'énoncé en - $h A n$ est précédé d'un énoncé décrivant l'état de fatigue de Juha (ligne 4) qui justifie son intention de rentrer. À la ligne 5, il s'agit d'ajouter à cette sensation subjective un autre fait qui relève des circonstances extérieures, indépendantes de la volonté de Juha (Ja olihan lauvantai «et après tout, on était samedi»). Le deuxième énoncé justificatif n'a pas pour fonction d'introduire dans le discours une information nouvelle, construite depuis la position $S_{0}$, mais d'y actualiser un état de choses $\mathbf{Z}$ préconstruit, relevant du repère virtuel $S_{X}$ et dont on peut penser ici qu'il fait partie des connaissances partagées, antérieurement établies. Introduit par le coordonnant ja «et», l'énoncé en - $h A n$ a pour effet de rejaillir sur l'énoncé précédent pour éliminer après coup l'idée qui pourrait en ressortir selon laquelle l'état physique (et moral) de Juha serait pour lui la seule raison de rentrer.

\subsection{Les unités complexes}

Nous avons soutenu l'idée que la relation prédicative évoquée dans les énoncés en $-h A n$ est préconstruite à l'extérieur de la position $\mathrm{S}_{0}$. Les exemples ont montré que la localisation de cette prédication préconstruite varie : elle peut être récupérée dans le contexte linguistique précédent, mais il peut s'agir aussi d'un «vouloir dire du monde» (cf. Paillard, 2011) qui s'impose à la conscience du locuteur, d'une contrainte émergeant des circonstances à laquelle le locuteur s'ajuste, ou encore d'une connaissance antérieurement établie, supposée partagée.

Notre dernière remarque porte sur les unités complexes où la relation prédicative préconstruite est introduite à l'aide d'un verbe de perception ou d'un verbe cognitif tel que näkyä/nähdä «être visible/voir», kuulua «être entendu», tietää «savoir», etc. La particule - $h A n$ est suffixée au verbe introducteur qui donne des indications sur l'accessibilité de l'état de choses $\mathbf{Z}$ exprimé dans la partie introduite, qui est grammaticalement soit une complétive en että «que», soit une construction participiale.

En (12a), il s'agit d'un verbe de perception à sens passif, näkyä «se voir/être visible» qui sert à introduire la mise en mots d'un état de choses $\mathbf{Z}$ («Juha arrive à avancer tout seul») observable en situation, dont Marja (au point de vue de laquelle 
le narrateur s'identifie) prend conscience soudainement et qui contraste avec ses attentes (cf. ex. 9). L'effet produit par l'énoncé en - $h A n$ est celui de surprise :

(12a)

Marja on lähtenyt mukana rantaa pitkin, noustakseen venheeseen suvannossa, ja auttaakseen sauvonnassa.

«Marja l'avait suivi le long de la rive pour embarquer sur l'eau calme et l'aider à ramer.»

3 Vaan näkyy-hän tuo pääsevän yksinkin. mais être visible-3-hAn celui-là arriver à avancer-PPR-GÉN seul-ADD «Mais il semble bien y arriver tout seul.»

$4 \quad$ Venhe nousee keveästi kiviä väistellen. Juhan selkä kumartuu ja oikenee yhä kiivaammin, vaikk'ei enää ole virrasta vastusta,

«La barque avance légèrement entre les pierres. Le dos de Juha se courbe et se redresse de plus en plus vite, bien qu'il n'y ait plus de courant contraire.» niinkuin pyrkisi kiireesti pois, pakoon. «On dirait qu'il se hâte de partir, qu'il fuit.» (Aho, Juha, 1911, XVII)

(12b)

$$
\begin{array}{lll}
\text { Vaan pääsee-hän } & \text { tuo } & \text { yksinkin. } \\
\text { mais arriver à avancer-3-hAn celui-là } & \text { seul-ADD } \\
\text { «Mais il y arrive tout seul.» } & &
\end{array}
$$

On peut remarquer tout d'abord que l'interprétation de l'énoncé en - $h A n$ ne changerait guère si on supprimait le verbe de perception (cf. $12 \mathrm{~b}$ avec - $h A n$ ajouté au verbe pääst ̈̈ «pouvoir avancer», placé en tête d'énoncé). Le verbe näkyä «être visible, se voir» a ici une valeur évidentielle qui est étroitement liée au fonctionnement du marqueur discursif $-h A n$ : il indique à quel titre est accessible l'état de choses $\mathbf{Z}$ qui est actualisé dans le discours en tant que préconstruit établi à l'extérieur de la position $\mathrm{S}_{0}$.

Le troisième élément qui contribue à créer l'effet d'un état de choses $\mathbf{Z}$ qui s'impose inopinément à la conscience de la locutrice est la forme sujet tuo «celuilà/il». Il s'agit d'un démonstratif d'éloignement qui, d'après Laury (1997, p. 7077), localise le référent à l'extérieur de la sphère du locuteur. Forsberg (2006, p. 84-94) qui s'intéresse à des cooccurrences entre - $h A n$ et tuo parle pour ce dernier d'une fonction évidentielle qui converge ici avec celle du verbe näkyä : tuo indique que l'énoncé s'appuie sur une observation directe de la part du locuteur. En combinaison avec $-h A n$, ce démonstratif implique une évidentialité directe : $-h A n$ associe la prédication au repère $S_{X}$ pour indiquer qu'elle n'est pas issue de prises de position subjectives, mais se trouve, au contraire, hors de leur portée (d'où l'idée d'une évidence), tandis que tuo donne au locuteur le rôle d'un témoin à la conscience duquel l'état de choses $\mathbf{Z}$ s'impose à l'instant même.

En (13a), l'unité complexe est formée à l'aide d'un verbe introducteur à sens

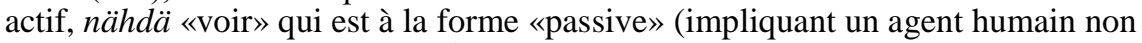
spécifié) et au temps parfait (onhan nähty «on a bien vu») : 
(13a)

1 Mutta yhä hän kuitenkin odotti, kun ei muutakaan osannut, odotti «Pourtant il [= Juha] attendait toujours, ne pouvant pas faire autrement. Il attendait»

2 pihasalla ollessaan, odotti ulkotöissä. Odotti kevätkalassa, aidanpanossa, «dans la cour, à son travail dans les champs, à la pêche, en posant une clôture,»

3 kasken hakkuussa, koko kevään pahimpain tulvainkin aikana. Ei tullut. «en déboisant l'essart, et au temps des grandes crues printanières. Elle [= Marja] n'arrivait pas.»

$4 \quad$ Mutta kesällä se varmasti tulee. Jos ei tule tänä kesänä, niin tulee toisena. «Mais elle viendrait certainement en été, si ce n'est pas celui-ci alors ce sera l'autre.» On-han nähty, että sotavuosinakin viedyt AUX-3-hAn voir-PPA.PAS CONJ année-guerre-PL-ESS-ADD qui est enlevé-PL «On avait bien vu des prisonniers» tulevat takaisin kymmenien vuosien päästä. (re)venir-3.PL ADV dizaine-PL.GÉN année-PL.GÉN bout-ÉLA «revenir des dizaines d'années après la guerre.

$7 \quad$ Sentähden pitää kaikki olla täällä sitä varten valmiina. «C'est pourquoi tout doit être prêt pour son retour.» (Aho, Juha, 1911, XIII)

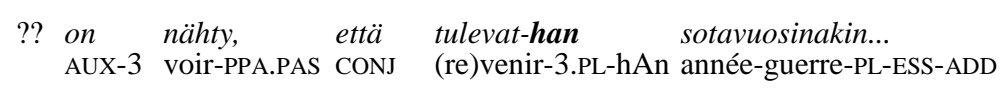

L'énoncé en - $h A n$ vient appuyer la conviction qu'a Juha sur la réalisation future de l'état de choses $\mathbf{Z}$, «Marja revient», exprimée dans les énoncés précédents (ligne 4). Sa valeur justificative repose sur le rapprochement du cas de Marja avec un autre cas comparable, celui des prisonniers de guerre. Le verbe introducteur on nähty «on a vu» indique que la prédication introduite est une connaissance antérieurement établie et supposée partagée. On peut noter ici que la préconstruction associée au repère virtuel $\mathrm{S}_{\mathrm{X}}$ à l'aide de $-h A n$ concerne la prédication complexe, c'est-à-dire que le verbe introducteur n'est pas dissociable du statut de préconstruit de la prédication introduite (cf. (13b) qui montre que le verbe introducteur doit se situer sous la portée de $-h A n)$. Le recours à - $h A n$ a pour effet de placer cet énoncé à valeur justificative à l'abri des mises en doute auxquelles sont exposés les énoncés à justifier construits depuis la position subjective $\mathrm{S}_{0}$ (ligne 4).

Les exemples (9)-(11), (12a) et (13a) montrent que la fonction du marqueur discursif $-h A n$ n'est pas liée au statut informationnel de l'énoncé : il peut s'agir aussi bien de l'actualisation dans le discours d'un état de choses nouveau pour le locuteur que du rappel d'une connaissance antérieurement établie. La notion de préconstruit, qui ne se confond pas avec ce que l'on entend par information 
connue $^{25}$ ou présupposition (cf. Caron, 2000, p. 12-13), a trait à la responsabilité énonciative qui est associée au repère virtuel $\mathrm{S}_{\mathrm{X}}$.

\section{CONCLUSION}

L'objectif de cet article a été d'examiner la fonction du marqueur discursif - $h A n$ en prenant comme point de départ son lien étymologique avec le logophorique hän «il/elle». Notre analyse s'est fondée sur l'hypothèse que dans le changement de catégorie, hän, tout en perdant sa référentialité et son autonomie en tant que mot, a conservé son sens indiciel, c'est-à-dire sa logophoricité. Dans la théorie de la grammaticalisation, on parle de retention pour désigner l'idée de maintien d'une partie des traits sémantiques de la forme source dans la forme à statut modifié (Bybee, Perkins \& Pagliuca, 1994, p. 15-19). Nous avons considéré la logophoricité comme le moteur même du développement qu'a connu hän.

Le phénomène de la logophoricité est habituellement défini en termes de coréférence : le logophorique utilisé dans le discours secondaire réfère au locuteur rapporté qui est défini dans le discours premier ; pour le dire autrement, le logophorique est coréférent avec le sujet d'un verbe du type «dire» (Roncador, 2006, p. 312 ; voir aussi Huang, 2006, p. 235 ; Boyeldieu, 2013). En nous appuyant sur la notion de scène énonciative, nous avons proposé de considérer que le propre du logophorique finnois hän est de situer son référent dans un espace énonciatif

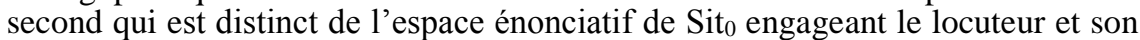
interlocuteur $^{26}$. La logophoricité de hän signifie alors que son origo énonciative $\left(\mathrm{S}_{\mathrm{X}}\right)$ se situe en dehors de l'espace intersubjectif de $\mathrm{Sit}_{0}$.

Le marqueur discursif $-h A n$ peut être rapproché typologiquement de la classe des marqueurs discursifs «du dire» qui indiquent que l'énoncé exprime un «Vouloir dire» qui n'est pas celui du locuteur (Paillard, 2011; Paillard \& Vu Thi, 2012)27. Partagent une identité sémantique abstraite avec le logophorique $h a ̈ n,-h A n$ rattache la relation prédicative de l'énoncé au repère virtuel $\mathrm{S}_{\mathrm{X}}$. Les effets de sens que produisent les énoncés en - $h A n$ dépendent de leur rapport avec les attentes qui s'associent aux positions subjectives $S_{0}$ et $S_{1}$ de la scène énonciative. Malgré leur diversité, les contextes où l'on trouve ce marqueur discursif se caractérisent tous par un conflit quelconque dans l'espace intersubjectif. Le double repérage énonciatif que $-h A n$ met en place, dans lequel la responsabilité énonciative du locuteur est réduite à l'actualisation dans le discours d'une prédication préconstruite, est un moyen de dépasser l'altérité des positions subjectives $S_{0}$ et $S_{1}$.

Enfin, cet article aura participé à une réflexion sur les énoncés à statut assertif atténué qui devrait être poursuivie dans une recherche ultérieure. Nous pensons tout particulièrement à l'agencement des constituants et à la prosodie des énoncés

25 Pour une discussion (déjà ancienne), voir Prince, 1981.

26 Rappelons que le terme logophorique, tel qu'il a été proposé par Hagège (1974, p. 290), signifie «renvoyant au discours».

27 Cette classe comporte notamment des marqueurs discursifs qui sont étymologiquement dérivés de verba dicendi (Paillard, 2011). 
en $-h A n$. On a vu que le point d'incidence de $-h A n$, toujours initial, peut ou non constituer un foyer de contraste, mais les conditions précises de cette variation restent à définir. À cela s'ajoute la description des traits suprasegmentaux qui aiderait à mieux circonscrire, dans une perspective typologique, ces énoncés qui ont l'apparence, mais pas le plein statut des assertions (interrogations ou injonctions ${ }^{28}$, selon le cas).

\section{ABRÉVIATIONS}

ABL ablatif ; ACC accusatif ; ADD particule additive ; ADE adessif ; ADV adverbe ; ALL allatif ; ANTILOG antilogophorique ; AUX auxiliaire ; CONJ conjonction ; ÉLA élatif ; ESS essif ; GÉN génitif ; ILL illatif ; IMP impératif ; INE inessif ; INF infinitif ; INTERJ interjection ; LOG logophorique ; NÉG verbe négatif ; NMLS marque de nominalisation ; NOM nominatif ; PAR partitif ; PAS passif (forme verbale impliquant un agent humain non spécifié) ; PEN particule énonciative ; PL pluriel ; POS suffixe possessif ; POT potentiel ; PPA participe passé ; PPR participe présent ; PRÉT prétérit ; TRA translatif ; Q suffixe interrogatif ; 1 première personne ; 2 deuxième personne ; 3 troisième personne.

\section{SOURCES DES EXEMPLES CITÉS}

Aho, J., 1911, Juha, Helsinki, Kustannusyhtiö Otavan kirjapaino.

Setälä, E. N., 1883, Lauseopillinen tutkimus Koillis-Satakunnan kansankielestä, Helsinki, Société de littérature finnoise.

SMS = Suomen murteiden sanakirja, tome 2, Helsinki, Kotimaisten kielten tutkimuskeskus ja Valtion painatuskeskus, 1988.

\section{RÉFÉRENCES BIBLIOGRAPHIQUES}

Banfield A., 1979, Où l'épistémologie, le style et la grammaire rencontrent l'histoire littéraire : le développement de la parole et de la pensée représentées, Langue française $\mathrm{n}^{\circ} 44$, p. 9-26.

Benveniste E., 1966, Problèmes de linguistique générale 1, Gallimard, Paris.

Bonnot, C., 2002, La portée des mots du discours : essai de définition (sur l'exemple du russe moderne), Cahiers de linguistique de l'INALCO ${ }^{\circ} 4$, p. 930.

Bonnot, C., 2004, Relation préconstruite et focalisation : pour une analvse unitaire des énoncés à accent non final en russe moderne, Études linguistiques et sémiotiques, Slovo vol. 30-31, Paris, Publications Langues O', p. 211-271.

Boyeldieu P., 2013, Introduction, in P. Boyeldieu (éd.), Logophorique et discours rapporté en Afrique centrale, Louvain-Paris, Peeters, p. 9-34.

Bybee J., Perkins R. \& Pagliuca W., 1994, The evolution of grammar: Tense, aspect, and modality in the languages of the world, Chicago, University of Chicago Press.

28 Pour l'emploi de - $h A n$ dans les énoncés injonctifs, qui n'a pas été examiné ici, voir ISK, 2004, § 831 . 
Caron, B., 2000, Assertion et préconstruit : topicalisation et focalisation dans les langues africaines, in B. Caron (éd), Topicalisation et focalisation dans les langues africaines, Louvain, Peeters, p. 7-42.

Culioli A., 1999, Pour une linguistique de l'énonciation. Domaine notionnel, tome 3, Paris, Ophrys.

Culy C., 1994, Aspects of logophoric marking, Linguistics 32, p. 1055-1094.

DeLancey S., 2001, The mirative and evidentiality, Journal of Pragmatics 33, p. 369-382.

De Vogüé S., 1987, La conjonction si et la question de l'homonymie, BULAG 13, Université de Besançon, p. 105-189.

De Vogüé S. \& Paillard D., 1987, Modes de présence de l'autre, Les particules énonciatives en russe contemporaine 2, Paris, Institut d'Etudes slaves, p. 1137.

Filippe-Deswelle C., 2014, Du réel à l'irréel : even if et la construction de tous les possibles, Travaux linguistiques du CerLico n²6, p. 191-205

Forsman Svensson P., 2003, Tuleppas siihen nenäilemään : Pragmaattiset liitepartikkelit 1600- ja 1700-luvun diskurssissa, in P. Forsman Svensson, Suomen kielen syntaksin kehitystä 1600-luvulta 1800-luvun alkupuolelle, Helsinki, Helsingin yliopiston kielikeskus, p. 52-82.

Forsberg, H., 1998, Suomen murteiden potentiaali : muoto ja merkitys, Helsinki, Société de littérature finnoise.

Forsberg H., 2000, The Finnic potential mood and the potential of Finnic, in J. Laakso (ed.), Facing Finnic : Some challenges to historical and contact linguistics, Helsinki, Finno-Ugrian Society and Department of Finno-Ugrian Studies of the University of Helsinki, p. 9-30.

Forsberg H., 2006, Modaalinen tuo, in T. Nordlund, T. Onikki-Rantajääskö \& T. Suutari (éds), Kohtauspaikkana kieli : Näkökulmia persoonaan, muutoksiin ja valintoihin, Helsinki, Société de littérature finnoise, p. 80-105.

Hagège C., 1974, Les pronoms logophoriques, Bulletin de la Société de Linguistique de Paris LXIX/1, p. 287-310.

Hakulinen A., 2001a, On some uses of the discourse particle kyl(lä) in Finnish conversations, in E. Couper-Kuhlen \& M. Selting (éds), Studies in interactional linguistics, Amsterdam, Benjamins, p. 171-198.

Hakulinen A., 2001b[1976], Liitepartikkelin -han/-hän syntaksia ja pragmatiikkaa, in L. Laitinen et al. (éds), Auli Hakulinen : Lukemisto. Kirjoituksia kolmelta vuosikymmeneltä, Helsinki, SKS, p. 44-90.

Hakulinen Auli \& Laitinen Lea 2008, Anaforinen nolla : Kielioppia ja affekteja, Virittäjä 112, p. 162-185.

Hakulinen L., 1979, Suomen kielen rakenne ja kehitys, Helsinki, Otava.

Hakulinen Lauri 1999[1951], Luennot suomen kielen partikkeleista, Helsinki, Helsingin yliopiston suomen kielen laitos.

Hanks W., 1992, The indexical ground of deictic reference, in A. Duranti \& C. Goodwin (éds), Rethinking Context. Language as an interactive phenomenon, Cambridge, Cambridge University Press, p. 43-76.

Huang Y., 2006, Anaphora, Cataphora? Exophora? Logophoricity, in K. Brown (éd.), Encyclopedia of Language and Linguistics, Second edition, vol. 1, Amsterdam, Elsevier, p. 231-237.

ISK = Hakulinen, A., Vilkuna, M., Korhonen, R., Koivisto, V., Heinonen, T. R. \& Alho, I., 2004, Iso suomen kielioppi, Helsinki, Société de la littérature finnoise, version électronique : http://scripta.kotus.fi/visk/etusivu.php/.

Kallio J., 1978, Kolmannen persoonan dialemmat, Sananjalka 20, p. 52-74. 
Kiuru, S., 1990, Mistä vanhaan kirjasuomeen tulivat jälkipronominit se ja hän?, Virittäjä 94, p. 278-306.

Kuiri K., 1984, Referointi Kainuun ja Pohjois-Karjalan murteissa, Helsinki, Société de littérature finnoise.

Laiho A., 1984, Liitepartikkelit ja niiden käyttö suomen kielessä : pääosin murrekorpukseen perustuva tutkimus, Mémoire de maîtrise, Université de Turku, Département de linguistique finnoise et générale.

Laitinen, L., 1995, Nollapersoona, Virittäjä 99, p. 337-358.

Laitinen L., 2002, From logophoric pronoun to discourse particle : A case study of Finnish and Saami, in I. Wischer \& G.Diewald (éds), New Reflections on Grammaticalization, Amsterdam, Benjamins, p. 327-344.

Laitinen L., 2004, Grammaticalization and standardization, in O. Fischer, M. Norde \& H. Perridon (éds), Up and Down the Cline : The Nature of Grammaticalization, Amsterdam, Benjamins, p. 247-262.

Laitinen L., 2005, Hän, the third speech act pronoun in Finnish, in R. Laury (éd.), Minimal reference: The use of pronouns in Finnish and Estonien discourse, Helsinki, SKS, p. 75-106.

Laitinen L., 2011, Kielitaiteilijat ja kielitieteilijät : Juhani Aho ja puhutun kielen kuunteleminen, in J. Nummi, R. Rossi \& S. Isomaa (éds), Pariisista Iisalmeen. Kansainvälinen ja kansallinen Juhani Aho, Helsinki, SKS, p. 153-185.

Larjavaara, M., 2007, Pragmasemantiikka, Helsinki, SKS.

Latvala S., 1895, Lauseopillisia havaintoja Luoteis-Satakunnan kansankielestä, Suomi III : 12, p. 1-89.

Latvala S., 1899, Lauseopillisia Muistoonpanoja Pohjois-Savon murteesta, Suomi III : 17 , p. $1-92$.

Laury R., 1997, Demonstratives in Interaction. The emergence of a definite article in Finnish, Amsterdam, Benjamins.

Leech G. N., 1987[1971], Meaning and the English Verb, London, Longman.

Lönnbohm O. A. F., 1879, Jääsken, Kirvun ja osittain Rautjärven ja Ruokolahden pitäjien kielimurteesta, Suomi II : 13, p. 1-163.

Makkonen M., 1967, Havaintoja hän-pronominin käytöstä suomen itämurteissa, Mémoire de maîtrise, Université de Helsinki.

Montaut A., 2006, Mirative Extensions of Aorist in Hindi/Urdu, in R. Singh (éd.), The Yearbook of South Asian Languages and Linguistics, Berlin/New York, Mouton de Gruyter, p. 71-86.

Nau N., 2006, Out of Africa: logophoric pronouns and reported discourse in Finnish and High Latvian dialects, Acta Linguistica Lithuanica LV, p. 55-87.

Ojansuu H., 1922, Itämerensuomalaisten kielten pronominioppia, Turku, Turun suomalaisen yliopiston julkaisuja.

Paillard, D., 1992, Repérage : construction et spécification, in La théorie d'Antoine Culioli: Ouverture et incidence, Paris, Ophrys.

Paillard D., 2009, Prise en charge, commitment ou scène énonciative, Langue française 162, p. 109-128.

Paillard D., 2011, Marqueurs discursifs et scène énonciative, in S. Hancil (éd.) Marqueurs discursifs et subjectivité, Publications des universités de Rouen et du Havre, p. 13-39.

Paillard D. \& Markowicz D., 1986, Le partage du savoir ou l'ignorance n'est pas un argument : à propos de la particule ved', Les particules énonciatives en russe contemporaine 1, Paris, Institut d'Études slaves, p. 89-123. 
Paillard D. \& Vu Thi N., 2012, Inventaire raisonné des marqueurs discursifs $d u$ français. Description. Comparaison. Didactique, Vietnam, Editions de l'Université nationale de Hanoï.

Peltola R., 2011, Cohésion modale et subordination : le conditionnel et le jussif finnois au miroir de la valeur sémantique et discursive du subjonctif français, Thèse de doctorat, Helsinki, Département de finnois et des langues et littératures finno-ougriennes et nordiques de l'Université de Helsinki, version électronique : <http://urn.fi/URN:ISBN:978-952-10-7376-2/>.

Philippe G., 1995, Embrayage énonciatif et théorie de la conscience : à propos de L'Etre et le Néant, Langages $\mathrm{n}^{\circ} 119$, p. 95-108.

Prince E. F., 1981, Toward a taxonomy of given - new information, in P. Cole (éd.), Radical Pragmatics, New York, Academic Press, p. 223-255.

Roncador von M., 1992, Types of logophoric marking in African languages, Journal of African Languages and Linguistics 13, p. 163-182.

Roncador von M., 2006, Logophoric pronouns, in K. Brown (éd.), Encyclopedia of Language and Linguistics, Second edition, vol. 7, Amsterdam, Elsevier, p. 312-315.

Seppänen E.-L., 1998, Läsnäolon pronominit. Tämä, tuo, se ja hän viittaamassa keskustelun osallistujaan, Helsinki, SKS.

Setälä, E. N., 1883, Lauseopillinen tutkimus Koillis-Satakunnan kansankielestä, Helsinki, Société de littérature finnoise.

Sirelius U. T., 1894, Lauseopillinen tutkimus Jääsken ja Kirvun kielimurteesta, Suomi III : 10, p. 1-143.

Stirling L., 1994, Logophoricity and long-distance reflexives, in R. E. Asher (éd.), The Encyclopedia of Language and Linguistics, vol. 4, Oxford, Pergamon, p. 2302-2306

Vilkuna M., 1989, Free Word Order in Finnish. Its Syntax and Discourse Functions, Helsinki, Société de littérature finnoise.

Välimaa-Blum R., 1987, The discourse function of the Finnish clitic -HAN: Another look, in P. Lilius \& M. Saari (éds), The Nordic Languages and Modern Linguistics 6. Proceedings of the sixth international conference on Nordic and General Linguistics in Helsinki, August 18-22, 1986, Helsinki, Helsinki University Press, p. 471-481.

Zribi-Hertz A., 1989, Anaphor binding and narrative point of view: English reflexive pronouns in sentence and discourse, Language 65, p. 695-727. 